\title{
LETTERS
}

The purpose of this Letters section is to provide rapid dissemination of important new results in the fields regularly covered by Physics of Plasmas. Results of extended research should not be presented as a series of letters in place of comprehensive articles. Letters cannot exceed four printed pages in length, including space allowed for title, figures, tables, references and an abstract limited to about 100 words. There is a three-month time limit, from date of receipt to acceptance, for processing Letter manuscripts. Authors must also submit a brief statement justifying rapid publication in the Letters section.

\section{A possible nature of "breathing" plasmas}

\author{
M. Z. Tokar \\ Institut für Plasmaphysik, Forschungszentrum Jülich GmbH, Association Euratom-FZJ, 52425, Jülich, \\ Germany \\ B. J. Peterson, Y. Nakamura, Y. Takeiri, and N. Noda \\ National Institute for Fusion Science, Toki-shi, Gifu-ken 509-5292, Japan
}

(Received 28 April 2000; accepted 31 July 2000)

A model for "breathing", plasmas observed in the large helical device [Y. Takeiri et al., Plasma Phys. Controlled Fusion 42, 147 (2000)] is proposed. It takes into account the synergism of radiation losses from both low- $Z$ (carbon, oxygen) and high- $Z$ (iron) impurities in the plasma power balance.

(C) 2000 American Institute of Physics. [S1070-664X(00)01411-7]

Recently, a phenomenon of "plasma breathing" has been observed in the large helical device (LHD): ${ }^{1,2}$ an increase of the electron density above a critical level led to self-sustained oscillations of diverse plasma parameters, e.g., integrated radiation or intensity of impurity spectral lines. Periodic variation of the ion saturation current discloses this phenomenon as repetitive acts of the plasma detachment from and reattachment to the divertor plates. In a detached phase the plasma becomes very cold not only in the divertor but in a significant part of the confined volume. This periodic expansion and contraction of the temperature profile led to the name "breathing."

It is well-known that a plasma detachment in tokamaks and stellarators can be caused by radiation from light impurities like carbon and oxygen when a critical density is achieved. ${ }^{3,4}$ However, to explain breathing one has to identify processes which account for the periodic reattachment of the plasma. Relevant models elaborated previously (see, e.g., Refs. 5 and 6) do not provide a firm explanation because they neglect a reaction of the plasma core on processes at the edge. In the present letter we discuss a breathing mechanism which takes into account the core response by considering the radiation losses from high- $Z$ ions. This approach was brought to mind by observations ${ }^{2}$ that breathing develops only when iron particles produced by erosion of divertor elements of stainless steel pollute the plasma additionally to "normal" light impurities. The mechanism proposed can be described as follows. In an attached state with a high enough temperature at the plasma edge there is an intensive erosion of the divertor plates and the density and radiation of iron from the plasma core increase in time. Consequently, the heat outflow to the plasma edge decreases and when it drops to a critical level a detachment owing to radiation of low- $Z$ impurities takes place. With reducing edge temperature the source of heavy impurity diminishes and its concentration in the core decreases due to outward transport of high- $Z$ ions. As a result the radiation losses from the plasma interim reduce and a reattachment occurs.

For a semiquantitative analysis we make some simplifying assumptions whose accuracy can be entirely proven only by a numerical modelling. The edge region where plasma heat conduction and line radiation of low- $Z$ impurities dominate the energy transport is assumed to be thin in comparison with the plasma minor radius. The plasma temperature $T$ is described here by the heat transport equation in a slab geometry: $:^{7,8}$

$$
-\kappa_{\perp} \frac{d^{2} T}{d x^{2}}=-n^{2} c_{l z} L_{l z}
$$

with the heat conductivity $\kappa_{\perp}$, electron density $n$ and fraction of low- $Z$ impurity, $c_{l z}$, assumed unchanging with the distance $x$ from the last closed magnetic surface (LCMS) toward the plasma core. The cooling rate of low- $Z$ impurities is computed in the "box" approximation: ${ }^{7,8}$

$$
L_{l z}\left(T<T_{l z}\right)=L_{l z}^{0} ; \quad L_{l z}\left(T>T_{l z}\right)=0 .
$$

The values $L_{l z}^{0}$ and $T_{l z}$ are determined by the impurity charge distribution. At the plasma edge this is governed by ionization and transport across magnetic surfaces, ${ }^{9}$ and $L_{l z}^{0} \approx 5$ $\times 10^{-7} \mathrm{eV} \mathrm{cm}^{3} \mathrm{~s}^{-1}, T_{l z} \approx 50 \mathrm{eV}$, for the impurities in question.

At the LCMS, $x=0$, we assume the boundary condition: ${ }^{10}$ 


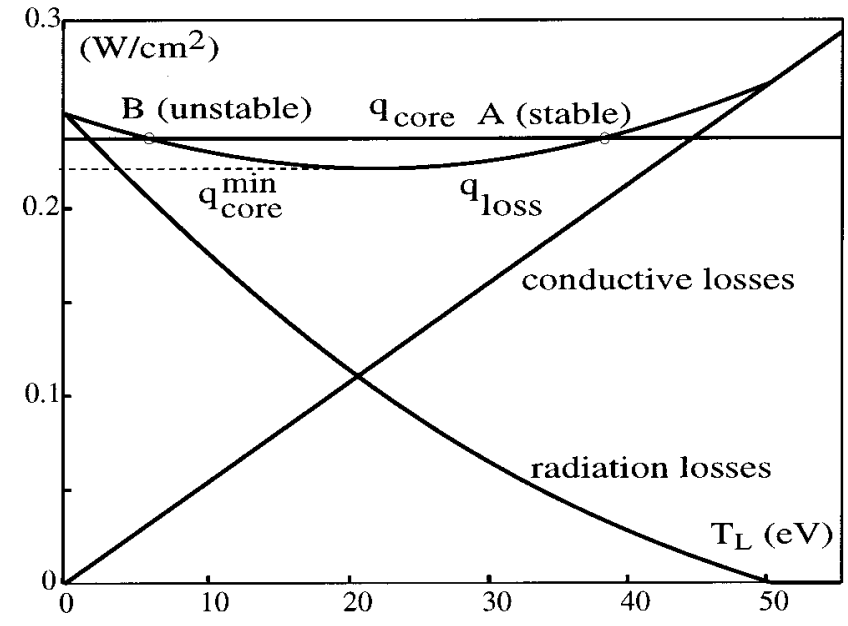

FIG. 1. Energy losses from the plasma and its stationary states.

$$
\frac{d T}{d x}=\frac{T}{\delta_{T}}
$$

with the $e$-folding length $\delta_{T}$ determined by the transport in the region with open field lines, i.e., in the stochastic zone and scrape-off layer. At the border of the plasma core, $x$ $=x_{\text {rad }}$, defined by the condition $T=T_{l z}$, the heat flux density, $q_{\text {core }}$, is prescribed.

Equation (1) is integrated analytically and the temperature at the LCMS, $T_{L}$, is governed by the balance between $q_{\text {core }}$ and the total energy loss from the edge with conduction and radiation, $q_{\text {loss }}=\kappa_{\perp} T_{L} / \delta_{T}+n^{2} c_{l z} L_{l z} x_{\text {rad }}$. A simple computation for $x_{\text {rad }}$ gives

$$
q_{\mathrm{loss}}=\sqrt{\left(\kappa_{\perp} \frac{T_{L}}{\delta_{T}}\right)^{2}+2 \kappa_{\perp} n^{2} c_{l z} \Lambda_{l z}}
$$

where $\Lambda_{l z}=L_{l z}^{0} \times \max \left(0, T_{l z}-T_{L}\right)$.

Figure 1 shows $q_{\text {loss }}$ and its conductive and radiative constituents versus $T_{L}$ for a typical plasma density of 7 $\times 10^{12} \mathrm{~cm}^{-3}$ in LHD discharges with breathing, ${ }^{1,2} \kappa_{\perp}$ $\approx 10^{17} \mathrm{~cm}^{-1} \mathrm{~s}^{-1}$ estimated from the measured temperature profile, $\delta_{T}=3 \mathrm{~cm}$ and $c_{l z}=1 \%$. With decreasing $T_{L}$ the conductive losses reduce while the radiation increases because the edge radiative layer becomes broader. This provides the minimum in $q_{\text {loss }}$. For $q_{\text {core }}>0.25 \mathrm{~W} / \mathrm{cm}^{2}$ there is a single stationary state where the input power is transported to the LCMS predominantly by conduction. When $q_{\text {core }}^{\min }$ $\approx 0.22 \mathrm{~W} / \mathrm{cm}^{2}<q_{\text {core }}<0.25 \mathrm{~W} / \mathrm{cm}^{2}$ there are two stationary states but only that with a higher temperature, $A$, is stable. ${ }^{10}$ The state $B$ is unstable: by a spontaneous decrease of $T_{L}$ the radiation losses grow stronger than the decrease of conductive losses and $T_{L}$ drops further. If the heat flux from the plasma core reduces to $q_{\text {core }}^{\min }$ there are no stationary states at all, the plasma edge cools down and a detachment occurs. ${ }^{10}$

In order to determine $q_{\text {core }}$ we consider the heat balance for the central plasma. After averaging over magnetic surfaces this is governed by the equation:

$$
\frac{1}{r} \frac{d}{d r}\left(-r \kappa_{\perp} \frac{d T}{d r}\right)=P-n^{2} c_{h z} L_{h z}(T),
$$

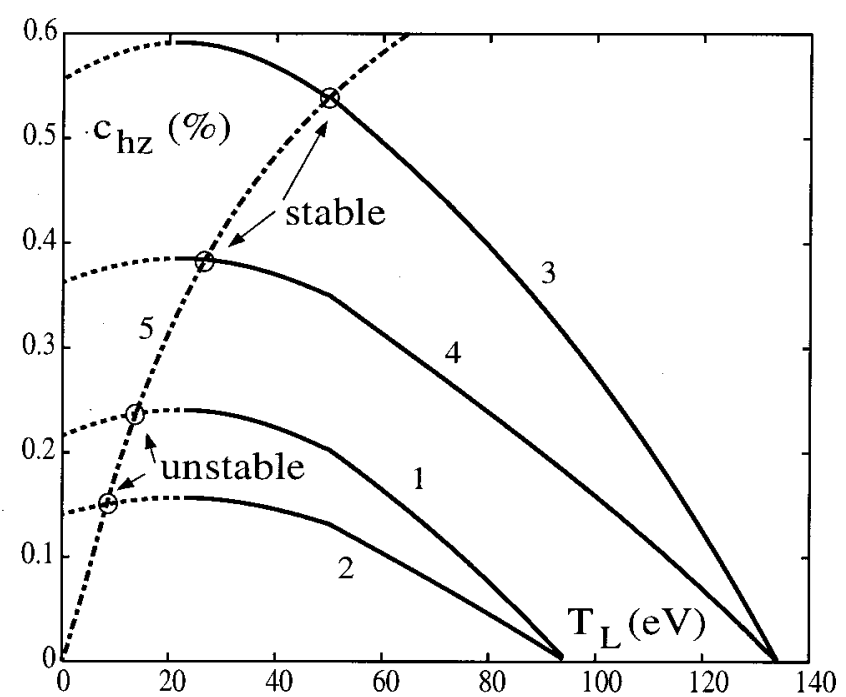

FIG. 2. Concentration of high- $Z$ impurity determined from the heat balance for the plasma parameters under breathing conditions with corona (curve 1) and noncorona (curve 2) models for high- $Z$ impurity and for a parameter $\Lambda_{\text {heat }}$ by a factor of 2 larger (curves 3 and 4, respectively) and from impurity particle balance (curve 5).

where $r$ is the effective minor radius of the surface computed by taking into account the three-dimensional (3D) magnetic geometry of the LHD, $P$ is the density of heating power, $c_{h z}$ and $L_{h z}$ the fraction and cooling rate of high- $Z$ impurity ions. Equation (3), multiplied by $r d r$ and integrated between the axis and the radius $r_{b}$ where the temperature reduces to $T_{b}$ $=\max \left(T_{l z}, T_{L}\right)$, results in

$$
q_{\text {core }}=\frac{W_{\text {tot }}}{4 \pi^{2} R r_{b}}-\frac{n^{2} c_{h z}}{r_{b}} \int_{0}^{r_{b}} Ł_{h z} r d r .
$$

Here $W_{\text {tot }}$ is the absorbed power, $R$ the major plasma radius, $n$ and $c_{h z}$ the volume averaged values. The integral above can be rewritten as $\int_{T_{b}}^{T_{c}} r(-d T / d r)^{-1} L_{h z} d T$, where $T_{c}$ is the temperature at the plasma axis. The measured temperature profile $^{1}$ can be roughly approximated by a parabolic one and $r(-d T / d r)^{-1} \approx r_{b}^{2} / 2 T_{c}$. Finally,

$$
q_{\text {core }} \approx n^{2} \frac{r_{b}}{2 T_{c}}\left(\Lambda_{\text {heat }}-c_{h z} \Lambda_{h z}\right)
$$

with $\Lambda_{\text {heat }}=W_{\text {tot }} T_{c} / 2 \pi^{2} R r_{b}^{2} n^{2}$ and $\Lambda_{h z}=\int_{T_{b}}^{T_{c}} \biguplus_{h z} d T$. For our qualitative analysis we assume according to Ref. $11 L_{h z}(T)$ $=L_{\text {cor }}(\alpha T)$, where $L_{\text {cor }}$ is the cooling rate in corona approximation. $^{12}$ For iron impurity with a typical $0.3<\alpha$ $<1, L_{h z}$ is much less at $T_{c} \approx 1 \mathrm{keV}$ than its maximum level achieved at $T \approx 70 \mathrm{eV} / \alpha$ and the $T_{c}$ dependence of $\Lambda_{h z}$ is weak.

Equating $q_{\text {loss }}$ and $q_{\text {core }}$ from Eqs. (2) and (4) we find a relation between stationary values of $T_{L}$ and $c_{h z}$. With the plasma heat conductivity estimated from the core thermal balance as $W_{\text {tot }} / 8 \pi^{2} R T_{c}$, this relation can be written as follows:

$$
c_{h z}=\frac{\Lambda_{\text {heat }}}{\Lambda_{h z}} \cdot\left[1-\sqrt{\left(\frac{r_{b} T_{L}}{2 \delta_{T} T_{c}}\right)^{2}+2 c_{l z} \frac{\Lambda_{l z}}{\Lambda_{\text {heat }}}}\right] .
$$

In Fig. 2 this dependence is shown for the LHD parameters: 
$r_{b} \approx 60 \mathrm{~cm}, R \approx 375 \mathrm{~cm}, W_{\text {tot }} \approx 450 \mathrm{~kW}$, with $L_{h z}$ in corona approximation and for $\alpha=0.5$. The dependences obtained with $\Lambda_{\text {heat }}$ by a factor of 2 larger are also presented; they correspond to discharge conditions of a smaller density or a higher power under which no breathing were observed. Thus if the concentration of high- $Z$ ions lies in the definite range the plasma heat balance allows two stationary states with different values of $T_{L}$. One of these states belongs to the dot part of the curves in Fig. 2 where $d q_{\text {loss }} / d T_{L}<0$ (see Fig. 1) and is unstable.

Additionally to the heat balance there is another interrelation between $c_{h z}$ and $T_{L}$ given by the particle balance for high- $Z$ impurity. The latter relates $c_{h z}$ with the impurity source due to erosion of divertor plates which is strongly dependent on $T_{L}$. The influx of sputtered iron neutrals through the LCMS is given by $J_{h z} \approx \Sigma_{j} Y_{j}\left(E_{j}\right) \cdot \Gamma_{j}$, where $Y_{j}$ and $\Gamma_{j}$ are the sputtering rate and outflow, respectively, of the background ions with different index $j$ corresponding to prefuelled helium, hydrogen from the neutral beam, carbon and oxygen impurities; the ion energy $E_{j} \approx \gamma_{j} T_{L}$ with the heat transmission factor $\gamma_{j}$ accounting for ion acceleration in the sheath potential near the plates. According to Ref. 13 $\Gamma_{j} \approx D_{\perp} \cdot\left(n_{j} / l_{j}\right)$ where $D_{\perp}$ is the diffusivity of charged particles, $n_{j}$ the core density of $j$ particles, $l_{j}$ the penetration depth of neutrals determined by the ionization cross-sections $\sigma_{j}: l_{j} \approx 1 / n \sigma_{j}$. The balance between $J_{h z}$ and outflow of high- $Z$ ions provides an additional relation between the concentration of the heavy impurity and $T_{L}$ :

$$
c_{h z}=\sum_{j} \frac{n_{j} \sigma_{j}}{n \sigma_{h z}} Y_{j}\left(\gamma_{j} T_{L}\right)
$$

with $\sigma_{h z}$ being the ionization cross section of iron atoms. This dependence is shown in Fig. 2 by the dot-and-dash line. The form of this curve is determined mainly by the temperature dependence of the sputtering rates ${ }^{14}$ which is very sharp in the vicinity of the threshold energy.

Equations (5) and (6) together determine an unique stationary state in which both the heat and impurity particle balances are satisfied. As one can see in Fig. 2 if the parameter $\Lambda_{\text {heat }}$ is larger enough this state is a one with a stable radiative layer at the plasma edge. This is in accordance with the observations that no breathing has been observed at a small density or a high power. For the conditions of plasma breathing the solutions of Eqs. (5) and (6) correspond to states which are unstable to the detachment at the plasma edge. If we put our system in such a stationary state the instability of the edge radiative layer brings the plasma either to the state $O_{1}$ or to $O_{2}$ (see Fig. 3). This occurs if the concentration of high- $Z$ impurity does not have enough time to change significantly during the development of this instability. This is really the case because the transport of high- $Z$ ions through the whole plasma requires much more time than cooling of the thin edge. If the system came, e.g., to the detached state $O_{1}$, the impurity source disappears and high- $Z$ impurity leave the plasma. When $c_{h z}$ drops down to the level $c_{h z}^{\min }$ the heat flux to the edge becomes large enough for the reattachment of the radiative layer, i.e., the plasma "jumps" from the state $A$ to the state $B$. Here the heavy

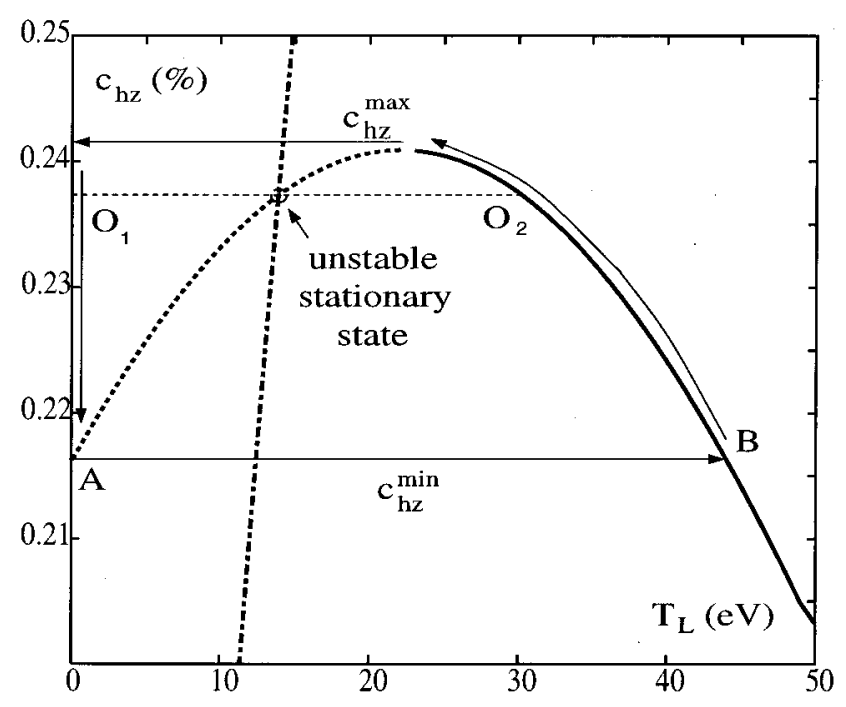

FIG. 3. Limit cycle corresponding to plasma breathing.

impurity source increases and $c_{h z}$ grows slowly to the level $c_{h z}^{\max }$ when detachment occurs again. Thus instead of a stationary state there is a limit cycle which corresponds to the plasma breathing. This behavior is analogous to oscillations in an electric circuit with a neon tube.

Transport of high- $Z$ impurity ions which determines the characteristic period of breathing is still poor understood theoretically for 3D magnetic configurations as in LHD. For a rough estimate we take into account only the diffusive component of impurity flow which clearly restricts the validity of the model. According to the transport analysis done for Wendelstein 7-AS ${ }^{15}$ a typical impurity diffusivity in stellarators is of $0.1\left(\mathrm{~m}^{2} / \mathrm{s}\right)$. This gives a characteristic diffusion time of $1 s$ in qualitative agreement with observations. A future numerical modelling should include also effects of such factors as the temperature gradient, radial electric field. There are many experimental evidences that these factors influence the particle transport significantly, e.g., through generation of a convective flux component.

${ }^{1}$ Y. Takeiri, Y. Nakamura, N. Noda et al., Plasma Phys. Controlled Fusion 42, 147 (2000).

${ }^{2}$ B. J. Peterson, Y. Nakamura, Y. Takeiri et al., Plasma Phys. Controlled Fusion (submitted).

${ }^{3}$ G. M. McCracken, J. Allen, K. Axon et al., J. Nucl. Mater. 145-147, 181 (1987).

${ }^{4}$ U. Samm, P. Bogen, H. A. Claassen et al., J. Nucl. Mater. 176-177, 273 (1990).

${ }^{5}$ S. I. Krasheninnikov et al., Nucl. Fusion 27, 1805 (1987).

${ }^{6}$ V. A. Abramov, S. G. Bespoludennov, Yu. L. Igitkhanov, and V. I. Pistunovich, J. Nucl. Mater. 176-177, 922 (1990).

${ }^{7}$ J. F. Drake, Phys. Fluids 30, 2429 (1987).

${ }^{8}$ K. Itoh and S.-I. Itoh, J. Phys. Soc. Jpn. 57, 1269 (1988).

${ }^{9}$ M. Z. Tokar, Nucl. Fusion 34, 853 (1994).

${ }^{10}$ M. Z. Tokar, Phys. Plasmas 7, 2432 (2000).

${ }^{11}$ Yu. N. Dnestrovskij and G. V. Pereversev, Nucl. Fusion 23, 633 (1983).

${ }^{12}$ D. E. Post, R. V. Jensen, C. B. Tarter et al., At. Data Nucl. Data Tables 20, 397 (1977).

${ }^{13}$ W. Engelhardt and W. Feneberg, J. Nucl. Mater. 76\&77, 518 (1978).

${ }^{14}$ W. Eckstein, J. Bohdansky, and J. Roth, Atomic and Plasma-Material Interaction Data for Fusion (Suppl. Nucl. Fusion, International Atomic Energy Agency, Vienna, 1991), Vol. 1, p. 51.

${ }^{15}$ R. Burhenn, A. Anton, J. Baldzuhn et al., Proceedings of the 8th International Toki Conference (NIFS, Nagoya, 1997), pp. 2-13. 